\title{
Construct Validation of Quality of Life for the Severely Mentally Ill
}

\author{
Gwendolyn Watkins J ohnson \\ Richard R. Bootzin
}

Aurelio J ose Figueredo

Michael R. Berren

\author{
Lee H. Sechrest \\ University of Arizona
}

\begin{abstract}
This study focused on the quality of life experienced by persons with severe mental illness (SMI). Previous studies indicate the need for a multi-dimensional approach to the study of quality of life and its subjective indicators. For the SMI, attention should be paid not only to the direct and intentional effects of interventions, but also to the indirect and unintentional effects, both negative and positive. Hence, a global evaluation of individuals within this group is indicated. A multitrait-multimethod approach to construct validation using confirmatory factor analysis was employed. The hypothesized factors were modeled as multiple traits and the multiple perspectives of the respondents (i.e. patient, case manager, family member) were multiple methods. A total of 265 severely mentally ill adults served by a network of agencies in four cities were randomly sampled. The sample was approximately 50\% male and 50\% female, ages ranged from 19 78 years.
\end{abstract}

Keywords: Structural Equation Modeling, Quality of Life, Severe Mental Illness (SMI), Multitrait-Multimethod (MTMM)

The definition and operationalization of the concept of quality of life (QOL) differs greatly from one study to another. Depending on the study, QOL may refer to the evaluation of certain domains of life, the subjective experience of the subject, client or patient, or to the evaluation of a given situation by others. QOL may also refer to a global evaluation, as in the current study, which focuses on the quality of life of the severely mentally ill (SMI).

The importance of defining and operationalizing QOL becomes clear when one considers that policy decisions are often based on improving QOL or some aspect of it. In this era of stiff competition for mental health funding, policy makers use information about functioning, well-being, and other important health outcomes to compare the costs and benefits of competing ways of financing and organizing mental health care services. This information may also be used by managers of mental health care organizations, who seek to produce the best value for each mental health care dollar. It is also utilized by clinical investigators for the purpose of evaluating new treatments and interventions, and providers striving to 
achieve the best possible client outcomes. The focus on outcomes of mental health care should be on the ability of clients to perform the daily activities of their lives, how they feel, and their own personal evaluation of their mental and general health (Stewart \&Ware, 1992).

Previous studies of the SMI (Slaughter, et al., 1991; Bootzin, et al., 1989) indicate the need for a multi-dimensional approach to the study of quality of life and its subjective indicators. This need is due to the complex nature of problems faced by these individuals and the multifaceted nature of interventions for them. For the SMI, consideration has to be given not only to the direct and intentional effects of interventions, but also to indirect and unintentional effects, both positive and negative.

\section{Theoretical Considerations}

A number of theoretical considerations were used as a basis for investigating QOL as it relates to the SMI. First, what are the subjective indicators of QOL? Second, what are the discriminable common factors or traits associated with QOL? What are the quantifiable relationships between these different facets of QOL? Is there a global higher-order common factor that underlies the various facets of QOL? Finally, what are the different respondent perspectives (methods) on patient QOL? What are the quantifiable relationships between respondent perspectives (methods)? Is there a global higher-order common factor representing some degree of consensus or agreement among the various respondentspecific perspectives?

To address these questions and several others that will be discussed later, this study employed a multitrait-multimethod (MTMM) approach to construct validation, using confirmatory factory analysis (CFA). The hypothesized factors are modeled as multiple traits, and the multipleperspective nature of the respondents (i.e. patient, case manager, family member) are multiple methods.

Mere examination of the MTMM matrix requires numerous judgment calls regarding the interpretation of values within the matrix including: 1) lack of specific criteria for evaluating the magnitudes of similarities and differences among the various elements of the MTMM matrix; 2) lack of specific criteria for selecting particular traits and methods for inclusion in the study; 3) inability of bivariate methods proposed by Campbell \& Fiske (1959) to analyze and separately estimate the components of variance (e.g., trait, method, and random variance) of which each "trait-method unit" is composed; and 4) difficulty in interpreting the results of the validation study when the assumptions of the MTMM method proposed by Campbell \& Fiske (1959) are not met.

The MTMM Factor Analytic Approach, as described by Figueredo, Ferketich, \& Knapp (1991) makes fully explicit the conceptual relations 
that are only implicit in the traditional bivariate analysis of the MTMM matrix. The various traits and methods of the MTMM matrix are more tangible as common factors than as abstract organizing principles defining the critical regions of the bivariate correlation matrix and specifying the theoretical interrelationships between them. The direct contributions of these latent traits and method constructs to the test scores and intercorrelations are more clearly identified and quantified. For example, whereas the traditional MTMM analysis could do little more than help to detect problems in discriminant validity, the CFA approach permits the identification of their causes by providing more specific diagnoses of the nature of these problems.

The application of CFA permitted a priori testing of hypotheses concerning specific theoretical interpretations as mentioned above. It was hypothesized that these data would yield seven lower-order common (trait) factors: psychopathology, satisfaction, psychosocial, self-care, home environment, physical/medical, and quality of life. This hypothesis was based on several studies that identified various domains of life as indicators of QOL and/or well-being: therapeutic services, symptomatology, social integration, affect, and psychosocial environment (Shadish, et al., 1985; Montgomery et al., 1987; Bootzin, et al., 1989); and symptoms, social skills, and housing quality (Slaughter, et al., 1991). All of these studies focused on the SMI. The Sickness Impact Profile (Gilson, et al., 1975), an instrument developed to measure outcomes of health care services in general, was also used to guide the identification of the hypothesized factors. The SIP focuses on the following domains: social interaction, ambulation or locomotion activity, sleep and rest activity, nutrition, usual daily work, household management, mobility and confinement, movement of the body, communication activity, leisure pastimes and recreation, intellectual functioning, interaction with family members, emotions, feelings and sensations, and personal hygiene.

The study also hypothesized the existence of a global higher-order QOL factor. If such a unitary higher order construct were confirmed, it would serve to unite the lower-order common (trait) factors. On the other hand, lack of convergence among these trait factors would yield seven discriminable factors, or facets of QOL.

The major goal of the study, from a statistical standpoint, was to develop a measurement model that can be used to formulate and test hypotheses regarding the relationship between the manifest or observed variables (subjective indicators) and the unobserved or latent variables (factors). Furthermore, this measurement model will be used to develop structural equations models that will causally relate the latent variables that have been factored from the observed variables (Long, 1983). 


\section{Method}

\section{Subjects}

Five percent of the population of adult severely mentally ill clients served by a network of agencies in four cities were randomly sampled. Sample sizes for each site were as follows: Tucson, 64; Albuquerque, 59; Akron, 80; and Providence, 62 (N=265). Subjects ranged from age 19 - 78 years. Selection criteria were based on the Arizona Checklist for Seriously Mentally III Determination. The seriously mentally ill are defined as those adult persons whose emotional or behavioral functioning is so impaired as to interfere with their capacity to remain in the community without supportive treatment. The mental impairment is severe and persistent and may result in a limitation of their functional capacities for primary activities of daily living, interpersonal relationships, homemaking, selfcare, employment, or recreation. Although persons with primary diagnoses of mental retardation or organic brain syndrome frequently have similar problems or limitations, they are not included in this definition.

\section{Data Collection}

Trained interviewers (graduate students and agency workers) collected data from the patients and, if possible, from a member of the patient's family. Patients were asked to provide the name of a family member who would give consent to be interviewed. During the interview, patients gave consent for the participation of their family member as well as consent for their own participation. Informed consent was also obtained for the patients' family members. Patients, their family members, and case managers were compensated for their participation. All data forms were coded to insure confidentiality.

\section{Measures}

A Patient Rating Guide (PRG), Therapist Rating Guide (TRG), and Family Rating Guide (FRG) were developed from items selected from a number of standard instruments with high reliability and validity (including the Colorado Client Assessment Record and the New York Functioning Scale). It was essential to assess outcomes that were relevant to the treatment of the SMI and at the same time to keep the measures brief so as not to tax the capacity of patients or family members. Item selection was based on the investigators' prior experience with measuring outcomes for SMI patients as part of the Arizona Pilot Project on 
capitation financing for the severely mentally ill (Bootzin, Berren, Figueredo, \& Sechrest, 1989). Case managers, who are advocates for the patients and track them through the mental health network, completed the TRG.

The rating guides are composed of Likert scales on which the respective respondents were asked to rate the extent to which the items were, at the present time, a problem for them (PRG), for the patient (TRG) and for their family member (FRG). The respondents were then asked to rate the same items as compared to the past, the past being one year ago. The patients and their family members were also asked to rate their level of satisfaction on several domains (patients were asked to rate living arrangements, necessities, and services; family members were asked to rate services only). Table 1 lists the QOL subscales and the respondent groups who completed each subscale.

Table 1

Subscales: Subjective Indicators of QOL

\begin{tabular}{lc}
\hline Subscale & Respondent(s) \\
\hline Psychopathology (PSYPAT) & P,C,F \\
Alcohol/ Drug (ALCDRU) & P,C,F \\
Hostility/ Aggression (HOSAGG) & P,C,F \\
Satisfaction with: & \\
$\quad$ Living Arrangements (SLIVARR) & $\mathrm{P}$ \\
$\quad$ Ability to Obtain Necessities (SNEC) & $\mathrm{P}$ \\
Mental Health services (SSVC) & $\mathrm{P}, \mathrm{F}$ \\
Psychosocial (PSYSOC) & $\mathrm{P}, \mathrm{F}, \mathrm{F}$ \\
Economics (ECON) & $\mathrm{P}, \mathrm{F}$ \\
Basic Needs (BASNEED) & $\mathrm{P}, \mathrm{F}, \mathrm{F}$ \\
Decision Making (DECMAK) & $\mathrm{C,F}$ \\
Basic Necessities (BASNEC) & $\mathrm{P}, \mathrm{C}, \mathrm{F}$ \\
Home Environment (HOMEN) & $\mathrm{C,F}$ \\
Living Arrangements (LIVARR) & $\mathrm{P}, \mathrm{C}, \mathrm{F}$ \\
Physical/Medical (PHYMED) & $\mathrm{C,F}$ \\
Quality of Life (QOL) & 37 \\
\hline Total number of subscales & 37
\end{tabular}

Note. $\mathrm{P}=$ Patient; $\mathrm{C}=$ Case Manager; F = Family Member

\section{Statistical Analyses}

Hierarchical analytical strategy. Since software limitations made it impossible to simultaneously analyze the individual items $(n=257)$ within a single multivariate model, a hierarchical analytic strategy consisting of five phases was used. The first three phases were essentially data reduction techniques. The final two phases were tests for convergence. SAS (SAS Institute, 1989) and EQS (Bentler, 1989) were used for the analyses. 
Phase 1. Item covariance matrices were computed and output by SAS using listwise deletion of missing data.

Phase 2. Items were rationally assigned to hypothesized subscale factor models and tested using confirmatory factor modeling performed by EQS. Items were tested for convergent validity, thus creating lower-order factors for the rationally derived subscales (Ferketich, Figueredo, \& Knapp, 1991; Figueredo, Ferketich, \& Knapp, 1991). The practical goodness-of-fit indices ranged from .96 to 1.00, indicating that these models were highly acceptable for practical purposes (Bentler \& Bonett, 1980). The chi-squared values for all models were statistically significant, indicating that the models do not perfectly fit all the covariances between the items.

Phase 3. Using the means of the standardized item scores for all nonmissing items on each subscale, SAS (PROC STANDARD and DATA) was used to compute unit-weighted common factor scores (Gorsuch, 1983) for all the subscales. Only those items found to be statistically significant were used to construct the subscale common factors.

Phase 4. Subscale covariance matrices were computed and output in SAS (PROC CORR), using mean substitution of missing data for each subscale and constructing a missing data dummy variable to control any systematic "missingness" bias. This procedure permitted the use of any remaining missing data (i.e., where any subject was missing all the validated indicators of a common factor) as potential information by allowing tests for any systematic deviation from the mean attributable to the "missingness" itself (Cohen \& Cohen, 1983), rather than uncritically ignoring such omissions as random occurrences. For example, a substantial number of subjects were missing all items within subscales relating to family member responses because they had no family available for the study. The missingness of the family member data represented a real difference in true subject status and not either an erratic refusal to answer the questions or any other such problems in measurement. The dummy variable, or "pseudovariate", was coded as equal to 0 for all cases for whom the data were missing and to 1 for all cases for whom the data were not missing. The dummy variable coding for this condition of "missingness" was entered into the measurement model and assessed for any systematic effects upon the indicators.

Phase 5. The final phase involved assigning the subscale common factors to lower-order constructs and testing them for convergent validity using confirmatory factor analysis via EQS. 


\section{Results}

\section{Theoretical Model}

Model 1 (Figure 1) represents the theoretical model as postulated. Thirty-seven subscale factors, as confirmed, are modeled as subjective indicators of QOL (Table 1). These subscale factors consists, on average, of seven items each. The seven trait factors (psychopathology, satisfaction, psychosocial, self care, home environment, physical/medical and quality of life) were allowed to freely correlate as were the three method factors (patient, case manager, family member).

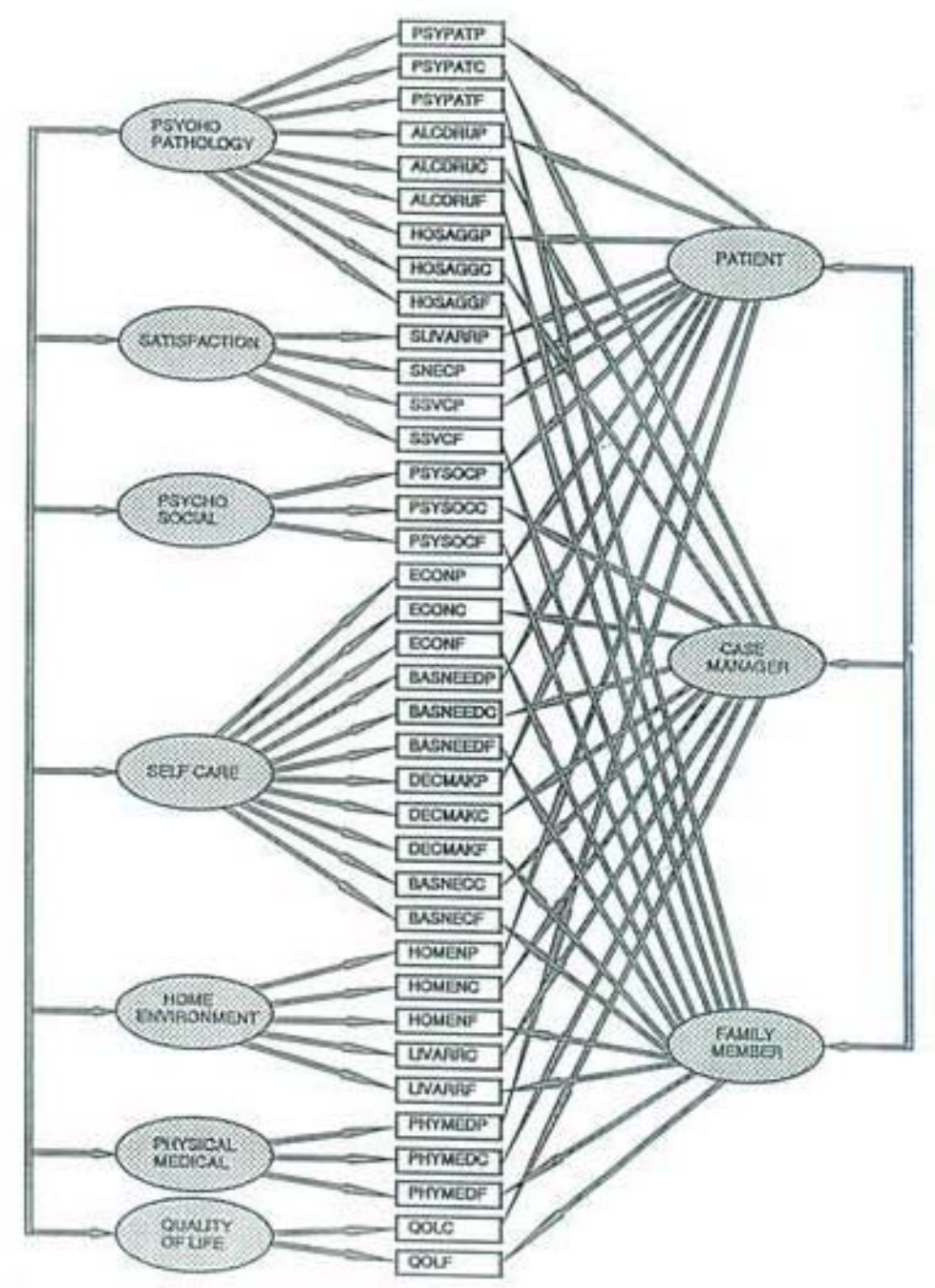

Figure 1: Theoretical Model 1 


\section{Lower-Order Common Factor Model}

Model 2 (Figure 2) displays the "inclusive" model, which is the initial model with all the paths, as originally hypothesized, included. Six lowerorder common trait factors were confirmed as follows: psychopathology, satisfaction, psychosocial, self-care, home environment, and physical/medical. The solid arrows indicate significant paths; the ghostly arrows indicate those paths that were found to be nonsignificant. There was not convergence for the lower-order quality of life factor. Three lowerorder common method factors were confirmed for the respondent perspectives (patient, case manager, and family member).

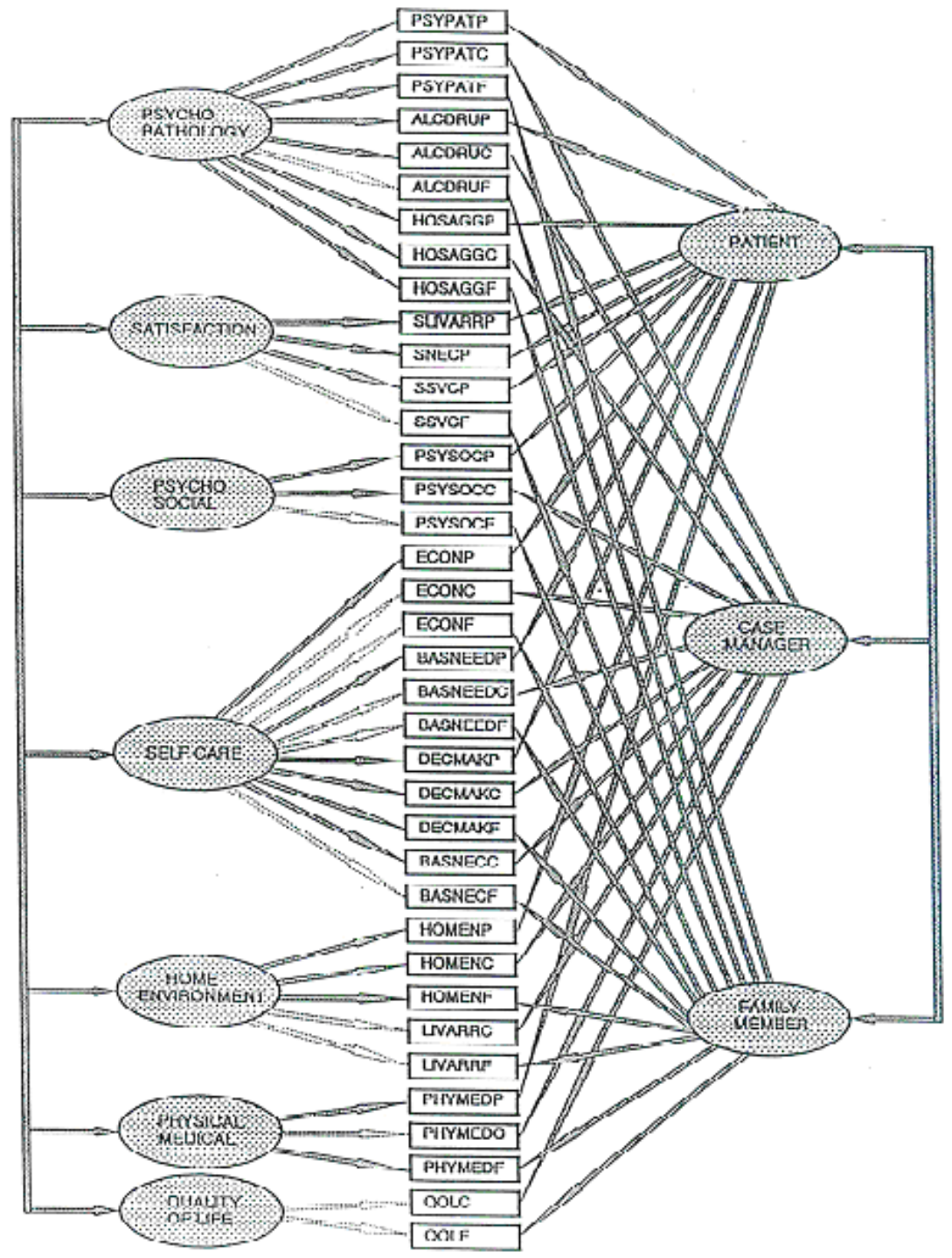

Figure 2: Model 2 - lower order common factors (inclusive model) 
The factor loadings and factor intercorrelations for Models 2-4 were very similar to those of the accepted model (Model 5) and will be discussed later.

\section{Higher-Order Common Factor Models}

A global higher-order common factor model, Model 3 (Figure 3) was used to test whether or not a unitary factor underlay the various facets (trait factors) of QOL. As noted above, the solid arrows indicate statistical significance, while the ghostly arrows indicate those paths that were

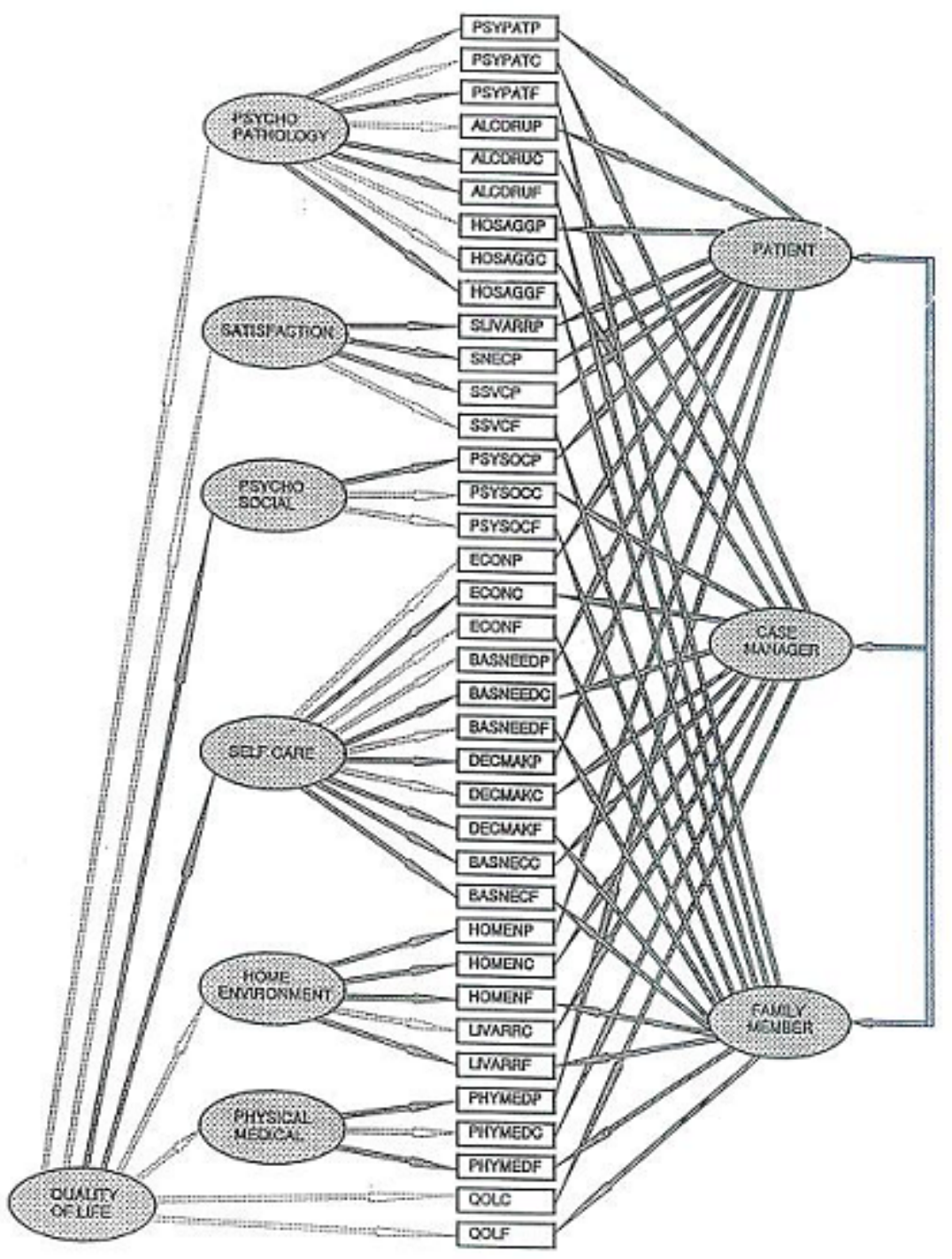

Figure 3: Model 3 - higher order common trait factor (QOL) 
nonsignificant. Only two of the trait factors converged on the hypothesized higher-order QOL factor. There was not a conceptual basis for determining what factor might unify the lower-order factors, psychosocial and selfcare. This model was conceptually rejectable as indicated by poor convergence among the trait factors.

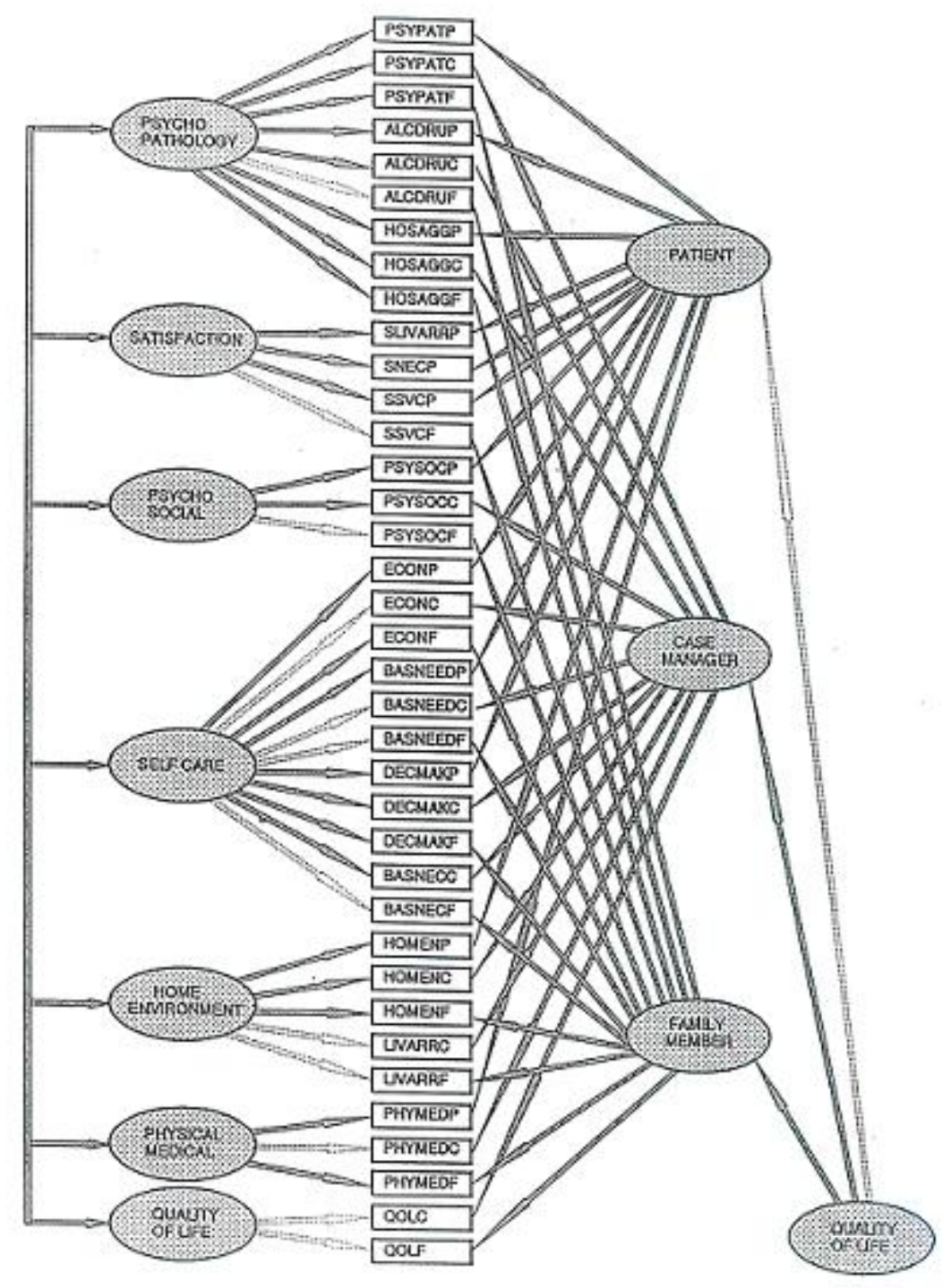

Figure 4: Model 4 - higher order common method factor

Model 4 (Figure 4) shows the same result when the existence of a global higher-order common factor was tested for the method factors. It was expected that some degree of consensus or agreement might exist between the respondents. Again, lack of convergence negates this notion. 
The convergence of the case manager and family member factors may, however represent a "nonself-report" factor.

\section{Dummy Variable Models}

Figures 5, 6, and 7 illustrate tests for any biases due to the missing family member data as discussed earlier. These tests allow for the estimation of effects of the patient having versus not having a family member available for the study. The 37 subjective indicators are the same

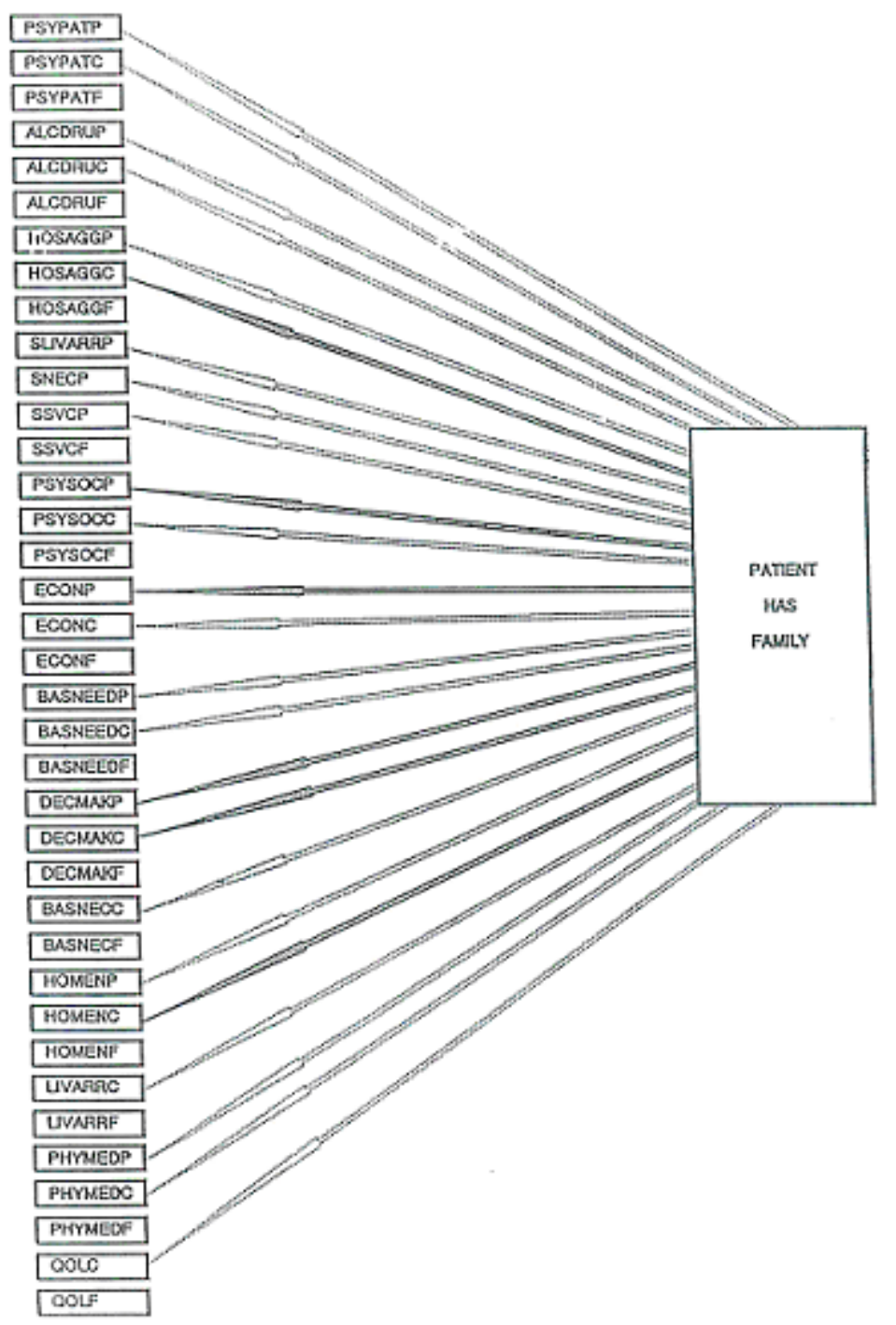

Figure 5: Model 5 - Models 5-7 test for "missingness" using a dummy variable. The solid arrows indicate significant pathways. 
as those displayed in Models 2 through 4 (a three dimensional representation of the entire model would position the dummy variable at 90 degree angles to the factors). Any effects, if present, would influence only the patient and case manager indicators. Figures 5 and 7 may be linked to Figure 2 to complete entire restricted models; however Figure 8 is to be paired with Figure 6 for a representation of the entire accepted

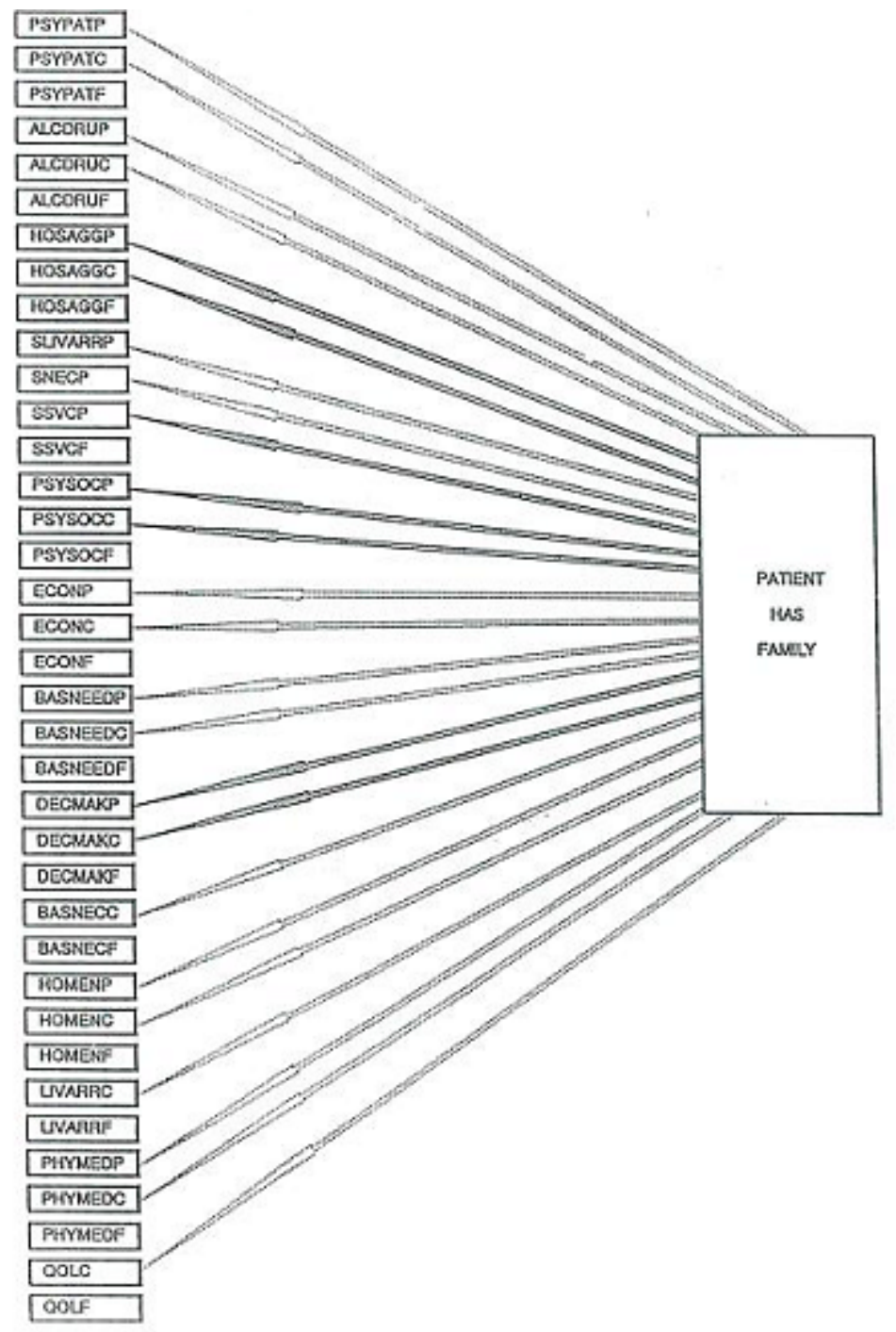

Figure 6: Model 6 - Test of "missingness" using dummy variable pairwise equality constraints imposed for nonself respondents. The solid arrows indicate significant pathways. 
model. The solid arrows indicate significant paths while the ghostly arrows indicate those paths that were found to be nonsignificant. The factor loadings and fit indices for these models are very similar to each other and will be discussed later as part of the discussion of the accepted model.

Figure 5 shows only 6 of the 24 patient/case manager indicators to be statistically significant. Those patients who had family members available for the study appeared to fare better than those patients who did not.

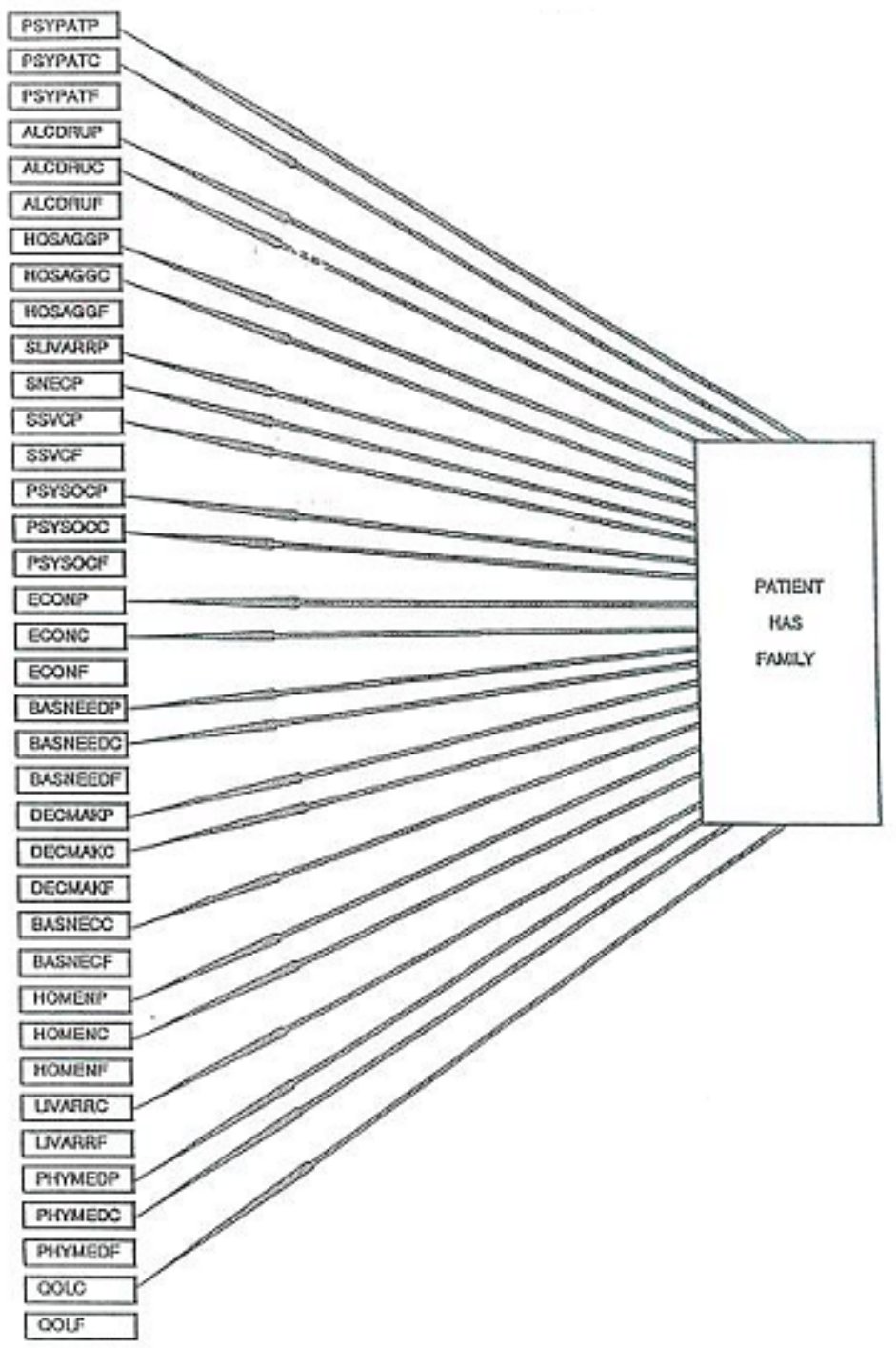

Figure 7: Model 7 - Test of "missingness" using dummy variable global equality constraints imposed. The solid arrows indicate significant pathways. 
These results suggest that for HOSAGGC, PSYSOCP, ECONP, DECMAKP, DECMAKC, and HOMENC, patients who did not have family members available show decreased QOL.

In order to test whether one perspective as opposed to another (patient and case manager) were equal, equality constraints were imposed across respondent pairs. For example, equality constraints were imposed across the indicators for psychopathology as reported by patient and case manager, for alcohol/drugs as reported by patient and case manager and so on; 9 such pairs and 6 single indicators were run in this model. Figure 6 shows that three pairs and one single indicator were found to be significant. For hostility/ aggression, psychosocial, and decision making as reported by patient and case manager and satisfaction with services as reported by the patient, patients' QOL seems to decrease at the same rate across the respondent pairs.

On the other hand, global equality constraints were imposed across all of the patient/case manager responses to test whether or not these respondents perspectives could be viewed as equal, that is whether QOL decreases at the same rate regardless of respondent. Figure 7 shows statistical significance across all of patient/case manager indicators, suggesting that patients' QOL appears to decrease at the same rate across respondents. Although this model was conceptually acceptable, it was statistically rejectable.

\section{Accepted Measurement Model}

Table 2 displays the statistical and practical indices of fit for all common factor measurement models. Although the $\chi^{2}$ values for all models were statistically significant, indicating that the models do not perfectly predict all the covariances between the subscales, the practical indices of fit (NNFl and $\mathrm{CFl}$ ) for all models were highly acceptable (Bentler \& Bonett, 1980). The "difference" tests are statistically nonsignificant for all models except Model 7, which is statistically rejectable, and not shown here. The difference tests are negligible for practical purposes, indicating that the "restricted" models (Models 3-4) perform nearly as well as the "inclusive" model (Model 2) in predicting the observed covariances. Based on the principle of parsimony, Model 8 (Figure 8) is the accepted measurement model. Models $3 \& 4$ were conceptually rejectable while Model 7 was statistically rejectable. 


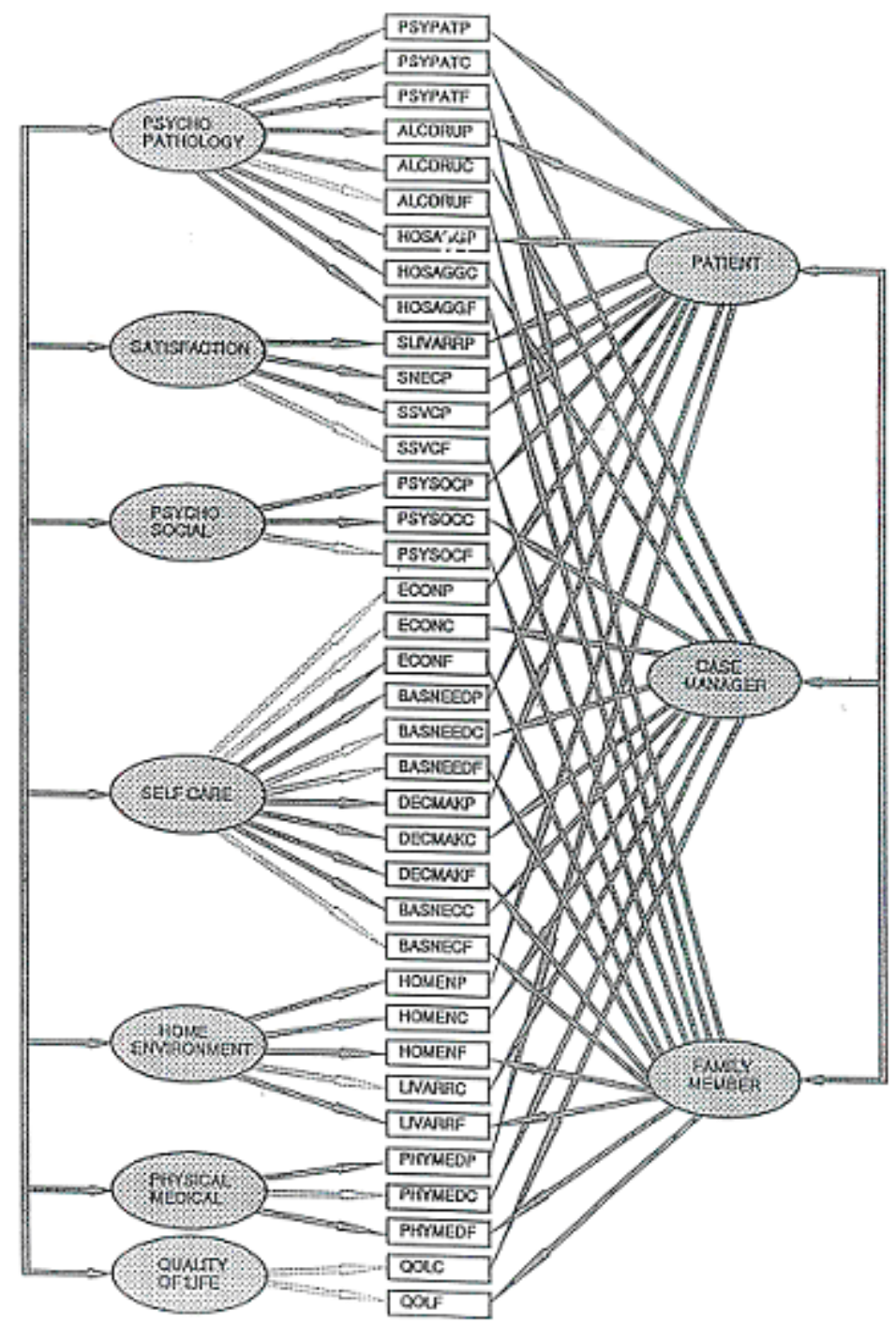

Figure 8: Model 8 - Accepted measurement model

The following factors were confirmed as in the previous restricted models: 37 subscale factors as subjective indicators of QOL, 6 trait factors, and three method factors. No higher-order QOL factor for either traits or methods was confirmed. 
Table 2

Statistical and Practical Indices of Fit for Factor Models

\begin{tabular}{lccccccc}
\hline Model & $\mathrm{df}$ & $\chi^{2}$ & NNFI & CFI & Model & $\Delta \mathrm{df}$ & $\Delta \chi^{2}$ \\
\hline $\begin{array}{l}\text { 2. Inclusive model } \\
\text { (No higher order factors) }\end{array}$ & 586 & $1012.00^{*}$ & .99 & .99 & & & \\
$\begin{array}{l}\text { 3. Trait HQOL factor } \\
\text { (No method HQOL factor) }\end{array}$ & 601 & $985.04^{*}$ & .99 & .99 & $3-2$ & 15 & -26.96 \\
$\begin{array}{l}\text { 4. Method HQOL Factor } \\
\text { (No trait HQOL factor) }\end{array}$ & 586 & $1011.96^{*}$ & .99 & .99 & - & - & - \\
\hline
\end{tabular}

Note: $*(\mathrm{p}<.001)$

Table 3 displays the factor loadings for the accepted model (factor loadings for the other restricted models are not included because they were extremely similar to those of the accepted model). The underscore denotes statistical significance. The heterogeneous nature (.002 to .929) of the trait factor loadings indicates sketchy convergent validity among the trait factors. The convergent validities on the trait factors are generally low and inconsistent across different respondents. The method factors, however display excellent convergent validity with statistically significant loadings across the board and generally high factor loadings.

Table 3 also displays the factor pattern for the "missing data indicator" dummy variable (V1) that is depicted in Figure 6. The factor pattern was essentially the same across the dummy variable models and is therefore only reported for the accepted model. The negative sign preceding the factor loadings indicates decreasing QOL. Significant effects of the "missing data" bias are few and weak (.034 to -.158); only three of the nine respondent pairs and one of the six single indicators were affected.

Table 4 shows the factor intercorrelations for the accepted model (the factor intercorrelations were extremely similar for all the models and are therefore only reported for the accepted model). The underscore denotes statistical significance. There is some evidence of discriminant validity among several of the trait factors as indicated by low significant correlations. The factor intercorrelations for the QOL factor (.80 to .34) are misleading in that they do not represent "real" effects. This illusion may be due to the fact there were only two indicators for this factor and those two indicators were poorly measured. The method factors are highly divergent as evidenced by the low correlations between them. There are significant but low correlations between patient/case manager and patient/ family member, but there is no correlation between case manager and family member. 
Table 3

Factor Loadings for Accepted Model (Model 8)

\begin{tabular}{|c|c|c|c|c|c|c|c|c|c|c|c|}
\hline Item & & & & Trait & & & & & etho & & \\
\hline & $\begin{array}{l}\text { PSY } \\
\text { PAT }\end{array}$ & SAT & $\begin{array}{l}\text { PSY } \\
\text { SOC }\end{array}$ & $\begin{array}{l}\text { SELF } \\
\text { CARE }\end{array}$ & $\begin{array}{l}\text { HOM } \\
\text { ENY }\end{array}$ & $\begin{array}{l}\text { PHY } \\
\text { MED }\end{array}$ & OOL & $P$ & C & $\mathrm{F}$ & V1 \\
\hline PSYPAT-P & .67 & & & & & & & .50 & & & -.08 \\
\hline PSYPAT-C & .18 & & & & & & & & .78 & & -.09 \\
\hline PSYPAT-F & .15 & & & & & & & & & .72 & \\
\hline ALCDRU-P & .32 & & & & & & & .36 & & & -.01 \\
\hline ALCDRU-C & .21 & & & & & & & & .58 & & -.01 \\
\hline ALCDRU-F & .09 & & & & & & & & & .38 & \\
\hline HOSAGG-P & .63 & & & & & & & .41 & & & -.15 \\
\hline HOSAGG-C & .16 & & & & & & & & .64 & & -.16 \\
\hline HOSAGG-F & .22 & & & & & & & & & .98 & \\
\hline SLIVARR-P & & .48 & & & & & & .39 & & & -.09 \\
\hline SNEC-P & & .60 & & & & & & .54 & & & -.06 \\
\hline SSVC-P & & .65 & & & & & & .23 & & & -.14 \\
\hline SSVC-F & & .05 & & & & & & & & .34 & \\
\hline PSYSOC-P & & & .93 & & & & & .34 & & & -.13 \\
\hline PSYSOC-C & & & .15 & & & & & & .79 & & -.13 \\
\hline PSYSOC-F & & & .03 & & & & & & & .73 & \\
\hline ECON-P & & & .28 & & & & & .60 & & & -.06 \\
\hline ECON-C & & & -.04 & & & & & & .72 & & -.06 \\
\hline ECON-F & & & .16 & & & & & & & .31 & \\
\hline BASNEED-P & & & .25 & & & & & .84 & & & -.08 \\
\hline BASNEED-C & & & .04 & & & & & & .89 & & -.07 \\
\hline BASNEED-F & & & -.01 & & & & & & & .58 & \\
\hline DECMAK-P & & & .89 & & & & & .44 & & & -.13 \\
\hline DECMAK-C & & & .12 & & & & & & .88 & & -.15 \\
\hline DECMAK-F & & & .19 & & & & & & & .59 & \\
\hline BASNEC-C & & & .13 & & & & & & .91 & & -.06 \\
\hline BASNEC-F & & & -.06 & & & & & & & .64 & \\
\hline HOMEN-P & & & & .43 & & & & .81 & & & -.04 \\
\hline HOMEN-C & & & & .36 & & & & & .80 & & -.04 \\
\hline HOMEN-F & & & & .24 & & & & & & .54 & \\
\hline LIVARR-C & & & & .00 & & & & & .69 & & -.11 \\
\hline LIVARR-F & & & & .17 & & & & & & .50 & \\
\hline PHYMED-P & & & & & .86 & & & .50 & & & -.10 \\
\hline PHYMED-C & & & & & .10 & & & & .77 & & -.09 \\
\hline PHYMED-F & & & & & .16 & & & & & .48 & \\
\hline QOL-C & & & & & .14 & & & & .76 & & -.03 \\
\hline QOL-F & & & & & .18 & & & & & .53 & \\
\hline
\end{tabular}

Note: $\mathrm{P}=$ Patient; $\mathrm{C}=$ Case Manager; $\mathrm{F}=$ Family Member; $\mathrm{V} 1=$ Dummy Variable for missing values; PSYPAT = Psychopathology; ALCDRU = Alcohol/Drugs; HOSAGG = Hostility/Aggression; SLIVARR = Satisfaction with Living Arrangements; SNEC = Satisfaction with Necessities; SSVC = Satisfaction with Service Arrangements; ECON = Economics; $\mathrm{PSYSOC}=$ Psychosocial; DECMAK = Decision making; BASNEED = Basic Needs; HOMEN = Home Environment; LIVARR = Living Arrangements; SNEC = Satisfaction with Necessities; PHYMED = Physical/Medical; QOL = Quality of Life 
Table 4.

Factor Intercorrelations for Accepted Model (Model 8)

\begin{tabular}{lrrrrrrrrrr}
\hline & \multicolumn{1}{c}{ Trait } & \multicolumn{1}{c}{ Method } \\
& 1 & 2 & 3 & 4 & 5 & 6 & 7 & $\mathrm{P}$ & $\mathrm{C}$ & $\mathrm{F}$ \\
\hline 1 Psychopathology & 1 & & & & & & & & \\
2 Satisfaction & .26 & 1 & & & & & & & \\
3 Psychosocial & .58 & .36 & 1 & & & & & & \\
4 Self care & .70 & .46 & .41 & 1 & & & & & \\
5 Home Environment & .05 & .19 & .12 & .38 & 1 & & & & \\
6 Physical/ Medical & .62 & .08 & .40 & .36 & -.15 & 1 & & & \\
7 QOL & .80 & .74 & .68 & .76 & .34 & .55 & 1 & & & \\
Patient & & & & & & & & 1 & & \\
Case Manager & & & & & & & & .24 & 1 & \\
Family & & & & & & & & .27 & .09 & 1 \\
\hline
\end{tabular}

\section{Discussion}

Evidence that quality of life is not well defined and operationalized is overwhelming. Review of the literature yields hundreds of studies across multiple disciplines concerning the concept of quality of life or some aspect of it. A few of these studies (Allen \& Bentler, 1985; Bremer \& McCauley, 1986) have reported QOL as a stable construct. For the vast majority of these studies, however, it appears that very little in the way of construct validation has been provided. Hence, QOL generally means many things to many people. In terms of policy/ decision making, program administration/ evaluation, and efficacy of treatment and interventions, the ability to accurately measure QOL is of paramount importance.

The findings of this study suggest that quality of life for the severely mentally ill is not a unitary higher-order latent common factor as postulated. Rather QOL is complex and multifaceted and is represented by six QOL facets or trait factors: psychopathology, satisfaction, psychosocial, self-care, home environment, and physical/medical. These factors and the quantifiable relationships between them may provide a comprehensive framework in which decisions about services to the SMI may be formulated. Since most SMI clients are exposed to multiple treatments and/or interventions and therefore experience multiple outcomes, it should be expected that their QOL will indeed be multifaceted.

The convergent validities on the QOL facets are, however, generally low and inconsistent across different respondents who might be expected to provide judgments (i.e., patient, case manager, and family member). This finding has implications for improving the measurement of these QOL facets. Great care should be taken in determining what questions to ask regarding QOL.

We call attention to the fact that failure to obtain responses from some family members and case managers did not affect the interpretations to be 
made of the data. Patients whose collaterals failed to provide data appeared to have generally lower quality of life, but not effect on the factor structure was evident.

Inconsistencies across the different respondent perspectives or method factors is illustrated by very high divergence among them. This high divergence is indicative of differing reports and suggests that these perspectives should not be used interchangeably. Specificity of the respondent is required when information regarding QOL is sought. These different perspectives appear to represent global or overall evaluations that are not specific to the various QOL facets. These evaluations may be value laden measures of satisfaction or dissatisfaction with respect to the QOL facets. The respondents, while evaluating the same facts, are probably evaluating them differently based on their own beliefs, values, etc. From a program evaluation standpoint, the implication is simple: Whom you ask is critical to what information you get.

It is clear that more empirical research is needed in order to better define and operationalize QOL for the seriously mentally ill. As the focus on outcomes of mental health care is increasingly geared toward the ability of the client to perform daily activities and how they feel about those activities, the need to better define QOL increases.

\section{Acknowledgements}

This research was supported by a grant from the National Institute of Mental Health (NIMH, Grant No. ROl MH43783-2P).

\section{References}

Allen, H. M. \& Bentler, P. M. (1985). Probing theories of individual well-being: A comparison of quality of life models assessing neighborhood satisfaction. Basic \&Applied Social Psychology, 6, 181-203.

Bentler, P. M. (1989). EQS: Structural equations program manual. Los Angeles, CA: BMDP Statistical Software.

Bentler, P. M., Bonett, D. G. (1980). Significance tests and goodness of fit in the analysis of covariance structures. Psychological Bulletin, 88, 588-606.

Bootzin, R. R., Shadish, W. R., \& McSweeney, A. J. (1989). Longitudinal outcomes of nursing home care for severely mentally ill patients. J ournal of Social Issues, 45, 31-48.

Bootzin, R. R., Berren, M., Figueredo, A. J . \& Sechrest, L. (1989). Caring for the chronically mentally ill: The Arizona Pilot Project. Invited Symposium. Arizona Psychological Association Conference, Tempe, Arizona.

Bremer, B. A. \& McCauley, C. R. (1986). Quality of life measures: Hospital interview versus home questionnaire. Health Psychology, 5, 171-177.

Campbell, D. T. \& Fiske, D. W. (1959). Convergent and discriminant validation by the multitrait-mu1timethod matrix. Psychological Bulletin, 56, 81- 105. 
Cohen, J ., \& Cohen, P. (1983). Applied multiple regression/ correlation analysis for the behavioral sciences. Hillsdale, $\mathrm{NJ}$ : Lawrence Erlbaum.

Ferketich, S. L., Figueredo, A. J ., \& Knapp, T. R. (1991). Focus on psychometrics: The multitrait-mu1timethod approach to construct validity. Research in Nursing \& Health, 14, 315-320.

Figueredo, A. J., Ferketich, S. L., \& Knapp, T. R. (1991). More on MTMM: The role of confirmatory factor analysis. Research in Nursing \& Health, 14, 387391.

Gilson, B. S., Gilson, J. S., Bergner, M., Bobbitt, R. A., Kressel, S., Pollard, W. E., \& Vesselago, M. (1975). The sickness impact profile: Development of an outcome measure of health care. American Journal of Public Health, 65, 1304-1310.

Gorsuch, R. L. (1983). Factor analysis. Hillsdale, NJ : Lawrence Erlbaum.

Long, J. S. (1983). Covariance structure models: An introduction to LISREL. Newbury Park, CA: Sage Publications.

Montgomery, L. M., Shadish, W. R., Orwin, R. G., \& Bootzin, R. R. (1987). Psychometric structure of psychiatric rating scale. Journal of Abnormal Psychology, 96, 167-170.

SAS Institute, Inc. (1989). SAS Language and Procedures: Usage, Version 6, First Edition. Cary, NC: SAS Institute.

Shadish, W. R., Orwin, R. G., Silber, B. G., \& Bootzin, R. R. (1985). The subjective well-being of mental patients in nursing homes. Evaluation and Program Planning, 8, 239-250.

Slaughter, J. G., Lehman, A. F., \& Myers, C. P. (1991). Quality of life of severely mentally ill adults in residential care facilities. Adult Residential Care J ournal, 5, 97-111.

Stewart, A. L. \& Ware, J . E. (1992). Measuring functioning and well-being: The medical outcomes study approach. Durham, NC: Duke University Press. 